\title{
Suprimento arterial dos intestinos do coelho da raça Nova Zelândia (Oryctolagus cuniculus)
}

\author{
Márcia Rita Fernandes Machado* \\ Sheila Viviane de Souza \\ Tatiana Catelan de Oliveira \\ Leandro Marcel Fernandes Cortellini \\ Roméria Rodrigues Barbosa \\ Faculdade de Ciências Agrárias e Veterinárias, Campus de Jaboticabal, UNESP \\ Via de Acesso Prof. Paulo Donato Castelanne s/n, CEP 14884-900, Jaboticabal - SP, Brasil \\ *Autor para correspondência \\ mrfmachd@fcav.unesp.br
}

\section{Resumo}

Estudaram-se a origem e a ramificação das artérias intestinais de coelhos da raça Nova Zelândia, mediante a injeção dos vasos arteriais com solução de látex e posterior dissecação de 20 espécimes adultos. A artéria mesentérica cranial origina-se da aorta abdominal e emite os seguintes vasos: ramos pancreáticos, artéria pancreaticoduodenal caudal; número variável de artérias jejunais; artéria ileocólica e ramos cólicos. A origem da artéria mesentérica caudal é próxima ao final da aorta abdominal e emite a artéria cólica esquerda que origina a artéria retal cranial e esta emite as artérias sigmóides.

Unitermos: artéria mesentérica cranial, artéria mesentérica caudal, intestinos, coelhos

\section{Abstract}

Arterial supply of the intestines of New Zealand rabbits (Oryctolagus cuniculus). The origin and ramification patterns of the intestinal arteries of rabbits were studied. The vessels were injected with a latex solution before dissection in 20 adult specimens. The cranial mesenteric artery originates from the abdominal aorta and gives off the following arteries: pancreatic branches; caudal pancreaticoduodenal; colic medium; jejunal (with variable number), ileocolic arteries, the colic branches, and ileal branches. The caudal mesenteric artery originates next to the end of the abdominal aorta and off a left colic artery that gives origin to the sigmoidal arteries and a cranial rectal artery.

Key words: cranial mesenteric artery, caudal mesenteric artery, intestines, rabbits 


\section{Introdução}

As funções primárias do aparelho digestório englobam a digestão e a absorção de nutrientes essenciais aos processos metabólicos dos animais, além de revelar hábitos alimentares gerais, auxiliando na classificação sistemática das espécies.

Segundo Morandini (1968), as artérias mesentéricas são os vasos responsáveis pelo suprimento sangüíneo do intestino delgado e grosso de répteis, aves e mamíferos, onde os nutrientes digeridos são absorvidos pelo epitélio e transferidos para a circulação (Hildebrand, 1995).

Para o rato (Greene, 1955; Habel e Stromberg, 1986) e para o camundongo (Cook, 1965), a artéria mesentérica cranial origina-se da porção abdominal da aorta, emitindo ramos para o pâncreas, duodeno e para diversas partes do intestino delgado e grosso, sendo seus ramos direcionados ao ceco e ao cólon; quanto à artéria mesentérica caudal, esta irriga partes do reto, e um de seus ramos anastomosa-se com um dos ramos da artéria mesentérica cranial.

Nos animais domésticos, a artéria mesentérica cranial origina-se caudalmente à artéria celíaca, com a qual forma em alguns casos um tronco. A artéria mesentérica caudal é um vaso ímpar que se origina próximo à divisão terminal da aorta, dividindo-se após um curto trajeto (Schwarze e Schröder, 1972; Ghoshal, 1981; Nickel et al., 1981).

Segundo Barone et al. (1996), a artéria mesentérica cranial do coelho emite a artéria cólica média, a artéria pancreático-duodenal caudal, a artéria cólica direita, um ramo ileocólico e, em um tronco comum, as artérias jejunais. Quanto à artéria mesentérica caudal, esses autores aludem ao fato de que este vaso se divide em artéria cólica esquerda e artéria retal cranial, da qual partem as artérias sigmóideas.

Em estudos com hamster dourado (Mesocricetus auratus), Orsi et al. (1975) afirmam que o arranjo dos ramos viscerais da aorta abdominal, destinados aos segmentos abdominais do aparelho digestivo, assemelha-se ao de outros mamíferos, tais como a cobaia, o coelho, o rato e o homem.
Nayar et al. (1983), ao observarem por arteriografia a anatomia das vísceras abdominais e da região lombar em caprinos, carnívoros, suínos e coelhos, relatam que a artéria mesentérica cranial, nestas espécies, origina os seguintes ramos: artéria cólica média, que é o ramo mais desenvolvido e este se anastomosa com a artéria gastroepiplóica esquerda; artéria ileocecocólica, a qual, nas cabras e no coelho, é o primeiro ramo emitido; artéria pancreático-duodenal cranial; e numerosas artérias jejunais que se anastomosam entre si, formando arcos. A artéria mesentérica caudal é um vaso pouco calibroso e se divide em ramos cranial e caudal.

Na paca, a artéria mesentérica cranial emite ramos para o pâncreas e a artéria cólica direita, que irriga o característico cólon "flutuante" destes animais; ainda da artéria mesentérica cranial emergem: a artéria cólica média, a artéria ileocólica, um grande ramo que segue para uma parte do cólon maior, a artéria ileocecocólica, que irriga o íleo, o ceco e o início do cólon maior, e uma série de ramos jejunais, ileais e cecais; nestes animais, a artéria mesentérica caudal se origina pouco antes da terminação da aorta e emite a artéria cólica esquerda, que se anastomosa com a artéria cólica média, além de ramos para o reto (Machado et al., 1996).

A artéria mesentérica cranial da cutia (Dasyprocta agouti) divide-se por bifurcação, trifurcação ou quadrifurcação, originando ramos às diversas porções do intestino e do pâncreas. A artéria mesentérica caudal bifurca-se vascularizando as porções finais do intestino grosso e reto. Anastomoses entre ramos originários da artéria mesentérica cranial e com os derivados da artéria mesentérica caudal também foram observados (Carvalho et al., 1999).

Considerando a importância econômica e experimental dos coelhos e a escassez de informações detalhadas sobre sua morfologia, objetivou-se descrever o suprimento arterial dos intestinos destes animais (raça Nova Zelândia), órgãos fundamentais no processo fisiológico de nutrição.

\section{Material e Métodos}

No desenvolvimento deste trabalho, foram utilizados 20 coelhos adultos (Oryctolagus cuniculus), da 
raça Nova Zelândia, machos, provenientes do Setor de Cunicultura da FCAVJ-UNESP.

Os animais, para o procedimento de injeção arterial, foram colocados em decúbito lateral direito e desta forma a injeção foi realizada mediante abertura do tórax, no $4^{\circ}$ espaço intercostal esquerdo, onde, após identificação, isolamento e canulação da aorta torácica no sentido caudal, o sistema arterial foi preenchido com solução de látex, corado e diluído.

Em seqüência, as peças foram fixadas em solução aquosa de formol a 10\%, por aproximadamente 72 horas. Após a fixação, procedeu-se a dissecação para identificação dos vasos em questão. Para a documentação foram realizadas fotografias.

\section{Resultados e Discussão}

Ao se comparar os achados sobre a origem e ramescência da artéria mesentérica cranial com os dados da literatura compilada, constatou-se que a artéria mesentérica cranial, de todos os coelhos da raça nova Zelândia observados, é um grande vaso que se origina da aorta abdominal (Figura 1), o que equivale às proposições de Greene (1955) e Habel e Stromberg (1986), para ratos; Schwarze e Schröder (1972), Ghoshal (1981) e Nickel et al. (1981), para os animais domésticos; Orsi et al. (1975), para o hamster dourado.

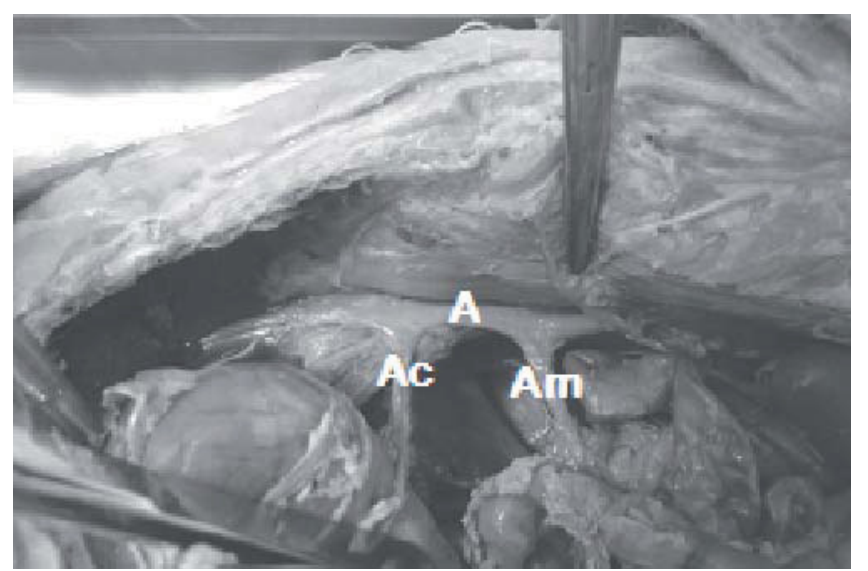

FIGURA 1: Fotografia da região cranial da cavidade abdominal de um coelho adulto, onde se observa a aorta abdominal (A) originando a artéria celíaca (Ac) e, caudalmente a esta, a artéria mesentérica cranial (Am).
Greene (1955), no rato, e Cook (1965), no camundongo de laboratório, ao descreverem o segundo ramo da aorta abdominal, denominaram-na de artéria mesentérica superior, ao invés de artéria mesentérica cranial, termo este que difere daquele preconizado pelo International Committee on Veterinary Gross Anatomical Nomenclature (1994), que registra artéria mesentérica cranial, nomenclatura adaptada neste trabalho.

Da mesma forma que Schwarze e Schröder (1972), Ghoshal (1981) e Nickel et al. (1981) relataram para os animais domésticos; Orsi et al. (1975) descreveram para o hamster dourado; e Habel e Stromberg (1986) para o rato, também se constatou, no coelho da raça Nova Zelândia, que a artéria mesentérica cranial é um vaso ímpar cuja origem é caudal à artéria celíaca (Figura 1).

Sobre a ramescência da artéria mesentérica cranial, semelhante às observações de Cook (1965), para o camundongo de laboratório, e às Carvalho et al. (1999), para a cutia, também se observou que este vaso emitia tanto ramos para o pâncreas e duodeno, quanto para diversas partes dos intestinos delgado e grosso, sendo um deles direcionado ao ceco e cólon; entretanto, essa ramificação e seu trajeto não foram identificados por Cook (1965) e Carvalho et al. (1999).

Por outro lado, neste trabalho as observações para os coelhos da raça Nova Zelândia assemelham-se, em parte, às descrições de Greene (1955), de Habel e Stromberg (1986), para ratos, de Barone (1996), para coelhos, de Orsi et al. (1975), para hamster, de Nayar et al. (1983), para caprinos, carnívoros, suínos e coelhos e de Machado et al. (1996) para pacas; pois apesar de no presente estudo se ter constatado que a artéria mesentérica cranial emitia as artérias pancreaticoduodenal caudal, cólica média, ileocólica e ramos jejunais (Figura 2), como registram estes autores, diferente dos relatos de Habel e Stromberg (1986), não se verificou a ocorrência de anastomose entre as artérias cólica média e caudal (Figura 2).

Também não se verificou, nas preparações analisadas, anastomose entre artéria gastroepiplóica esquerda e cólica média, tal como citaram Nayar et al. (1983), para coelhos e carnívoros, tampouco observaram-se 
vários ramos irrigando o pâncreas, conforme citaram Machado et al. (1996), para pacas.

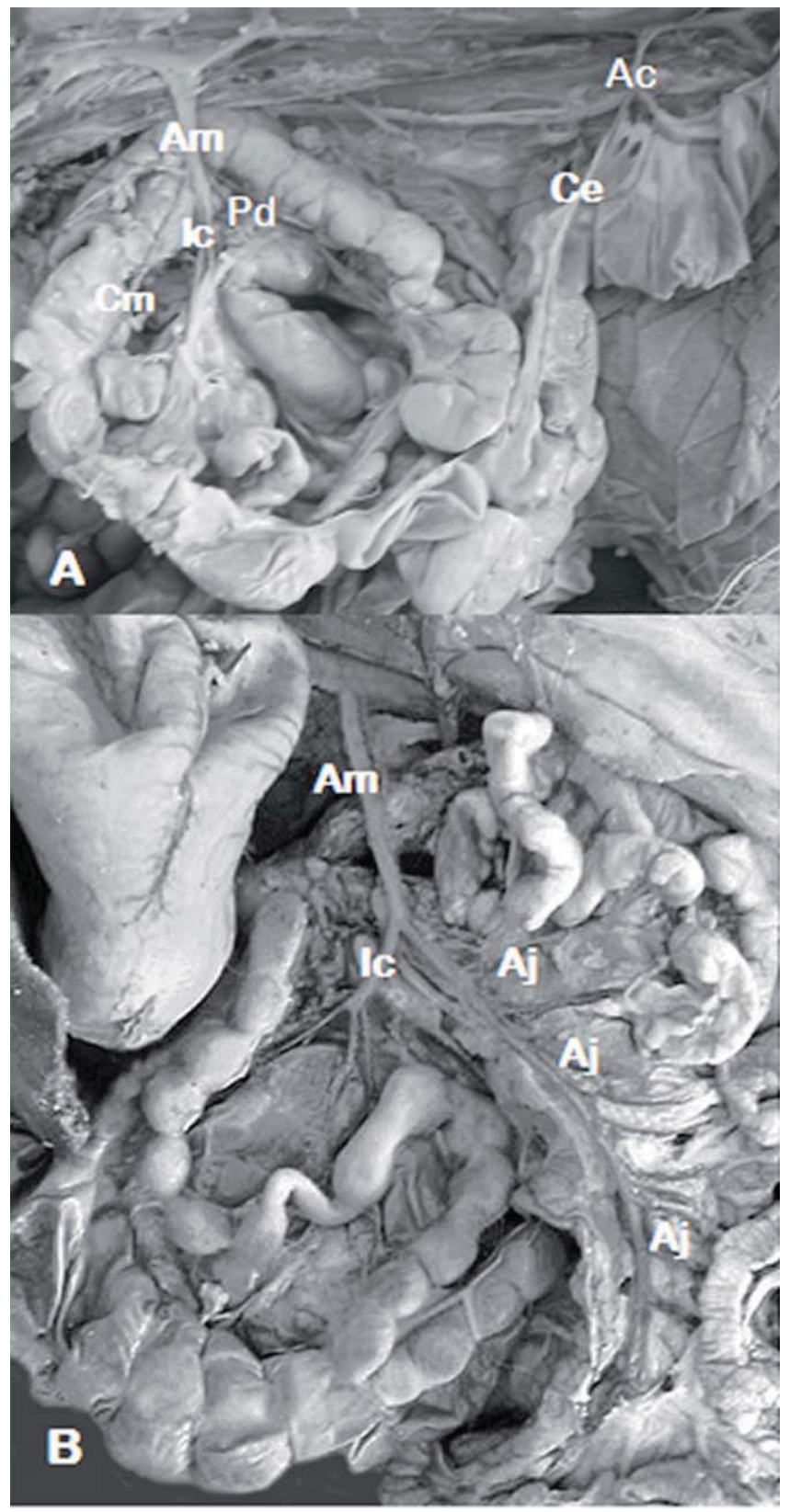

FIGURA 2: Fotografias da região cranial da cavidade abdominal de coelhos adultos onde, em A se observa a artéria mesentérica cranial (Am) originando a artéria pancreaticoduodenal caudal (Pd), a artéria cólica média (Cm), a artéria iliocólica (Ic). Também se nota a artéria mesentérica caudal (Ac) originando a artéria cólica esquerda (Ce) que se anastomosa com a artéria cólica média $(\mathrm{Cm})$. Em B se observa a artéria mesentérica cranial (Am) originando a artéria ileocólica (Ic) e as artérias jejunais $(\mathrm{Aj})$.

Conquanto Nayar et al. (1983) descreveram que a artéria ileocecocólica surgia como primeiro ramo da artéria mesentérica cranial, observou-se nos coelhos da raça Nova Zelândia a ocorrência da artéria pancreática duodenal como primeiro ramo da mesentérica cranial; também não se constatou a presença do ramo ileocecocólico, como aludiram Nayar et al. (1983) e Machado et al. (1996), ainda que estes últimos autores relatassem que, na paca, tanto a artéria ileocólica quanto a artéria ileocecocólica eram ramos da mesentérica cranial.

Averiguou-se a presença de 12 a 22 artérias jejunais originando-se da artéria mesentérica cranial, achados estes semelhantes às descrições de Nayar et al. (1983), para as espécies que estudaram, e aos relatos de Machado et al. (1996) para a paca, apesar destes autores não citarem o número de vasos, por eles observados. Por outro lado, essas afirmações diferem dos relatos de Orsi et al (1975) para hamster, uma vez que estes autores citam a ocorrência de aproximadamente sete ramos jejunais.

A artéria mesentérica caudal do coelho da raça Nova Zelândia, observada, é um vaso ímpar e pequeno que se origina próximo ao término da aorta abdominal e se divide, após um curto trajeto, em artéria cólica esquerda e artéria retal cranial, da qual partem as artérias sigmóideas. Estes achados corroboram com as descrições de Schwarze e Schröder (1972); Orsi et al. (1975); Ghoshal (1981); Nickel et al. (1981); Habel e Stromberg (1986); Nayar et al. (1983); Barone (1996) e Machado et al. (1996), todavia Nayar et al. (1983) não fizeram alusão sobre a ramificação desse vaso, afirmando que, no rato, os ramos viscerais da aorta abdominal apresentavam variações importantes, sem detalhá-las.

Pôde-se constatar que, em $100 \%$ das amostras analisadas, não houve variação no padrão de ramificação tanto da artéria mesentérica cranial como da artéria mesentérica caudal, e que este padrão se assemelha ao dos mamíferos em geral.

\section{Agradecimento}

Os autores agradecem à Fundação de Amparo à Pesquisa do Estado de São Paulo (FAPESP) pela bolsa de iniciação científica (processo ${ }^{\circ} 00 / 01001-0$ ) concedida. 


\section{Referências}

Barone, R. 1996. Artères. In: Barone, R. (Ed.). Anatomie Comparée des Mammifères domestiques Esplancnologie. Éditions Vigot, Paris, France, p.327-347.

Carvalho, M. A. M.; Miglino, M. A.; Dio, L. J. A. Melo, A. P. F. 1999. Artérias mesentéricas cranial e caudal em cutias (Dasyprocta aguti). Journal of Veterinary Science Federal University of Uberlândia, 5 (2): 17-24.

Cook, J. M. 1965. Circulatory System. In: Cook, J. M. (Ed.). The Anatomy of the Laboratory Mouse. Academic Press, London, England, p.87-88.

Ghoshal, N. G. 1981. Coração e artérias. In: Getty, R (Ed.). Anatomia dos Animais Domésticos. 5. ed. Interamericana, Rio de Janeiro, Brasil, p.560-562.

Greene, G. C. 1955. Circulatory System. In: Greene, G. C. (Ed.) Anatomy of mamimals. Transactions of the American Philosophical, New York, USA, p.177-235.

Habel, R.; Stromberg, M. W. 1986. Anatomy and embryology of the laboratory rat. Biomed Verlag, Wörthsee, Germany, 270pp.

Hildebrand, M. 1995. Análise da estrutura dos vertebrados. Atheneu, São Paulo, Brasil, 263 pp.

International Committee on Veterinary Gross Anatomical Nomenclature. 1994. Nomina Anatomica Veterinária. $4^{\text {th }}$ ed. World Association on Veterinary Anatomist, New York, USA, 198pp.
Machado, M. R. F.; Nogueira, T. M. R.; Miglino, M. A.; Artoni, S. M. B.; Araújo, M. L.; Gianonni, M. L. 1996. Ramificação da artéria mesentérica cranial e artéria mesentérica caudal da Paca (Agouti paca, Linnaeus, 1766). Anais do Congresso Brasileiro de Anatomia, Ceará, Brasil, p.29.

Morandini. C. 1968. Zoologia. 2. ed. Nobel, São Paulo, Brasil, 1196pp.

Nayar, K. N. M.; Singh, G.; Singh, Y. Singh, A. P.; Singh, G. R. 1983. Comparative arteriographic anatomy of the abdominal viscera and lumbar region in goats, dogs, pigs and rabbits. Indian Journal of Animal Sciences, 53 (12): 1310-1419.

Nickel, R.; Schummer, A.; Seiferle, E.; Sack, W. O. 1981. The circulatory system, the skin, and the cutaneous organs of the domestic mammals. Verlag Paul Parey, Berlin, Germany, 588pp.

Orsi, A. M.; Pinto e Silva, P.; Mello-Dias, S.; Oliveira, M. C. 1975. Considerações sobre a remescência da aorta abdominal do hamster dourado (Mesocricetus auratus). Revista Brasileira de Pesquisas Médicas e Biológicas, 8 (5-6): 459-462.

Schwarze, E.; Schröder, L. 1972. Compendio de Anatomia Veterinaria: Aparato Circulatório y Piel. Acribia, Zaragoza, Espanha, $247 \mathrm{pp}$. 